40



RESEARCH ARTICLE

\title{
Experience Verification of 63 Cases of Liver Transplantation Anesthesia Management
}

\author{
Haruko Hasegawa ${ }^{1, *}$, Makoto Ozaki ${ }^{1}$, Sumire Yokokawa ${ }^{1}$, Yoshihito Kotera $^{2}$ and Hiroto Egawa ${ }^{2}$ \\ ${ }^{\prime}$ Department of Anesthesiology, Tokyo Women's Medical University, Tokyo, Japan \\ ${ }^{2}$ Department of Gastroenterology, Tokyo Women's Medical University, Tokyo, Japan
}

\begin{abstract}
:
Background:

We reviewed the intraoperative management of previous liver transplantation (LT) cases to identify an optimal anesthetic method, which may affect patient outcomes and lead to faster postoperative recovery for future recipients.

Methods:

This single-center retrospective study reviewed 63 patients who underwent LT, including 51 living donor LT (LDLT), seven deceased donor LT (DDLT), and five simultaneous liver-kidney transplantation patients. We examined the patients' backgrounds, intraoperative management (anesthetic method, water balance, and catecholamine dosage), and postoperative courses (hospitalization period, length of intensive care unit stay, renal function).

Results:

All patients received general anesthesia using inhalational anesthetics, either sevoflurane or desflurane, and both drugs were administered similarly. Rocuronium was administered at its usual dose despite liver failure. All patients undergoing preoperative dialysis due to acute kidney injury were successfully withdrawn from dialysis after surgery. The albumin infusion volume was $32 \%$ of the total infusion and transfusion volume. The five-year survival rate was $88 \%$ and graft failure occurred in one case.

Conclusion:

The anesthetic management of LT is currently conducted empirically in our institution, and we could not identify an optimal anesthetic method However, we drew some conclusions. First, the use of human atrial natriuretic peptide as a drug infusion and appropriate transfusion management was expected to restore renal function. Second, the infusion volume of albumin was high. Third, the usual dose of rocuronium was required because excessive bleeding may cause unstable plasma drug concentration. Our results will be useful in future multi-institutional studies or metaanalyses and further improving the outcomes of future transplant recipients.
\end{abstract}

Keywords: Intraoperative anesthesia management, Liver transplant, Colloidal solutions, Human atrial natriuretic peptide, Single-center retrospective study, Postoperative care.

\begin{tabular}{|l|l|l|l}
\hline Article History & Received: February 22, 2021 & Revised: August 10, 2021 & Accepted: August 16, 2021 \\
\hline
\end{tabular}

\section{INTRODUCTION}

Liver transplantation (LT) was first introduced in the US in 1963 by Starzl et al., following which it came into practice in Europe. In Japan, Deceased Donor Liver Transplantation (DDLT) was performed for the first time in 1964 and Living Donor Liver Transplantation (LDLT) in 1989. Since then, the

* Address correspondence to this author at Department of Anesthesiology, Tokyo Women's Medical University, 8-1 Kawada-cho, Shinjuku-ku, Tokyo 162-8666, Japan; Tel: +810333538111 ; Fax: +810333592517 ;

E-mail: haru555888@hotmail.co.jp post-transplant prognosis has gradually improved through years of accumulated experience with surgical procedures, intraoperative and postoperative management, control of immunosuppressants, and a further improvement in scientific knowledge and techniques.

Currently, LT is performed in $>400$ patients in Japan each year [1]. Intraoperative anesthesia management is complicated, as coagulopathy causes heavy bleeding and infusion is difficult due to hypoalbuminemia. Hemodynamics also tends to be unstable. With the recent improvement in survival rates of LT 
recipients, assuring the postoperative quality of life of patients is important. Thus, based on previous cases, anesthesiologists must investigate the methods of intraoperative management that may affect patient outcomes and lead to faster postoperative recovery.

Accordingly, we aimed to identify an optimal anesthetic method and postulate a set of perioperative guidelines that could be used as the standard when administering anesthesia to LT patients. Our results can be applied across multiple institutions and will result in a thorough examination of the current aspects of anesthetic management, eventually improving the outcomes of future recipients.

\section{METHODS}

This study was conducted retrospectively on LT recipients who were treated by the surgical and anesthesiology teams of our institution. We examined the current anesthetic approaches, such as specific anesthetic methods, anesthetic agents, and monitoring methods. A total of 63 patients aged $>18$ years who had received DDLT, LDLT, and Simultaneous Liver-kidney Transplantation (SLKT) and were managed by the Department of Anesthesiology at Tokyo Women's Medical University between January 1, 2014, and December 31, 2018, were included in our study. We chose these dates because our computer-based anesthesia charts were upgraded in 2014. We extracted the patients' background data, various physical and blood test data, hospitalization periods, length of stay in the Intensive Care Unit (ICU) and High Care Unit (HCU), and postoperative courses (infection, introduction of dialysis, reoperation, etc.) from the hospital's electronic medical records. We also collected data on the method of anesthesia, operative time, anesthesia duration, blood loss, urine volume, in-out balance, and catecholamine dosage from the computerbased anesthesia charts (Mirrel of Fukuda Denshi, Tokyo). We used our data to examine the intraoperative management methods and postoperative courses. For postoperative infection, we extracted the data of patients who had clinical signs of infection and required active treatment by way of antimicrobial agents. The patients' backgrounds, anesthesia method, and postoperative courses served as primary outcomes, and the characteristics of patients with long ICU and HCU stays served as secondary outcomes.

\section{RESULTS}

A total of 63 patients underwent LT at the Tokyo Women's Medical University Hospital over the last five years. Of these, 51 underwent LDLT, eight underwent DDLT, and four underwent SLKT (from a deceased donor). The five-year survival rate for LDLT recipients was $88 \%$.

\subsection{Preoperative Evaluation}

Each patient's background is summarized in Table 1. The average age of LDLT patients was $48 \pm 13$ years, DDLT was $45 \pm 12$, and SLKT was $46 \pm 5$. The Model for End-stage Liver Disease (MELD) score was $18 \pm 6$ (LDLT), 18.6 \pm 5 (DDLT), with SLKT patients having the highest score $(19 \pm 3)$. Coagulability on the day before surgery tended to be low in DDLT patients, mild in LDLT patients, and almost standard value in SLKT patients.

Preoperative eGFR was relatively well-maintained in LDLT patients $(71 \pm 39)$, while it showed a slight decrease in DDLT patients $(52 \pm 22)$. The percentages of patients undergoing preoperative dialysis were $11 \%$ in LDLT and $14 \%$ in DDLT, and dialysis was performed in all SLKT patients. Of the seven LDLT patients on preoperative dialysis, five patients had chronic renal failure and two patients had an acute renal failure due to hepatorenal syndrome. The causes of liver disorders are shown in Table 2.

Primary Biliary Cholangitis (PBC) was the most common in LDLT patients (19\%), alcoholic and drug-induced injuries were most common in DDLT patients ( $28 \%$ each), and Autosomal Dominant Polycystic Kidney Disease (ADPKD) was the most common primary disease in SLKT patients. In many of these ADPKD patients, liver function was maintained, but infection of the hepatic cysts was uncontrollable.

Table 1. Patient demographics and clinical characteristics.

\begin{tabular}{|c|c|c|c|}
\hline Variable & LDLT & DDLT & SLKT \\
\hline Total number & 51 & 7 & 5 \\
\hline Male, N(\%) & $24(47 \%)$ & $2(28 \%)$ & $2(40 \%)$ \\
\hline Female, N(\%) & $27(52 \%)$ & $5(71 \%)$ & $3(60 \%)$ \\
\hline Age (years) & $48(13)$ & $45(12)$ & $46(5.6)$ \\
\hline BMI $\left(\mathrm{kg} / \mathrm{m}^{2}\right)$ & $22.3(3.5)$ & $24.5(2.7)$ & $21(2.3)$ \\
\hline MELD score & $18.9(6)$ & $18.6(5)$ & $19.2(3)$ \\
\hline Preoperative PT-INR & $1.47(0.3)$ & $1.8(0.3)$ & $1.1(0.1)$ \\
\hline Preoperative APTT (second) & $47.7(17)$ & $55(11)$ & $44.3(18)$ \\
\hline Preoperative Platelet count $\left(\times 10^{4}\right)$ & $11.0(8.3)$ & $8.6(5)$ & $14.6(7.5)$ \\
\hline Preoperative HbAlc $(\%)$ & $4.8(1.0)$ & $5.0(1.5)$ & $4.8(0.7)$ \\
\hline Preoperative eGFR (ml/minute/1.73m $\left.\mathrm{m}^{2}\right)$ & $71(39)$ & $52.9(22)$ & $15(13)$ \\
\hline Hemodialysis, N(\%) & $6(11 \%)$ & $1(14 \%)$ & $5(100 \%)$ \\
\hline Diabetes, N(\%) & $7(13 \%)$ & $1(14 \%)$ & $0(0 \%)$ \\
\hline Smoking & - & - & - \\
\hline
\end{tabular}


(Table 1) contd.....

\begin{tabular}{|c|c|c|}
\hline Variable & LDLT & DDLT \\
\hline Ex-smoker, N(\%) & $14(30 \%)$ & - \\
\hline Non-smoker, N(\%) & $25(54 \%)$ & $2(40 \%)$ \\
\hline Unknown, N(\%) & $5(9 \%)$ & - \\
\hline
\end{tabular}

Values are represented as mean (standard deviation) or number of patients (\%).

LDLT: living donor liver transplantation, DDLT: deceased donor liver transplantation, SLKT: simultaneous liver-kidney transplantation, BMI: body mass index, PT-INR: prothrombin time international normalized ratio, APTT: activated partial thrombin time, HbA1c: hemoglobin A1c, eGFR: estimated glomerular filtration rates

Table 2. Etiology of liver failure.

\begin{tabular}{|c|c|c|c|}
\hline Primary Disease & LDLT & DDLT & SLKT \\
\hline Total number & 51 & 7 & 5 \\
\hline PBC & $10(19 \%)$ & - & - \\
\hline PSC & $4(7 \%)$ & - & $1(20 \%)$ \\
\hline Alcoholic cirrhosis & $5(9 \%)$ & $2(28 \%)$ & - \\
\hline Congenital biliary atresia & $3(5 \%)$ & $1(14 \%)$ & - \\
\hline Drug-induced & $5(9 \%)$ & $2(28 \%)$ & - \\
\hline Budd-Chiari syndrome & $3(5 \%)$ & - & - \\
\hline NASH & $5(9 \%)$ & - & - \\
\hline $\mathrm{HCV}$ & $5(9 \%)$ & $1(14 \%)$ & - \\
\hline $\mathrm{HBV}$ & $3(5 \%)$ & - & - \\
\hline ADPKD & $3(5 \%)$ & $1(14 \%)$ & $3(60 \%)$ \\
\hline Hyperoxaluria & - & - & $1(20 \%)$ \\
\hline Unknown & $5(9 \%)$ & - & - \\
\hline
\end{tabular}

Values are represented as number of patients (\%).

LDLT: living donor liver transplantation, DDLT: deceased donor liver transplantation, SLKT: simultaneous liver-kidney transplantation, PBC: primary biliary cirrhosis, PSC: primary sclerosing cirrhosis, NASH: nonalcoholic steatohepatitis, HCV: hepatitis C virus, HBV: hepatitis B virus, ADPKD: autosomal dominant polycystic kidney disease

\subsection{Intraoperative Management}

Intraoperatively, we monitored the electrocardiogram, noninvasive blood pressure, invasive arterial blood pressure (FloTrac $^{\mathrm{TM}}$ sensor), central venous pressure (Presep, Edwards Lifescience, USA), pulse oximeter, exhaled gas concentration, and body temperature (through bladder catheter). We used the Bispectral Index monitor (BIS monitor, Vista A-3000 ${ }^{\mathrm{TM}}$, NIHON KODEN, Tokyo, Japan) or the Patient State Index (PSI, Sedline ${ }^{\circledR}$, Masimo, USA) for electroencephalogram reading. We used Transesophageal Echocardiography (TEE) in $22 \%$ of patients and measured pulmonary arterial pressure in $3 \%$ of patients.

The intraoperative anesthetic management is summarized in Table 3. The operative time was $682 \pm 114$ minutes for LDLT, $690 \pm 113$ minutes for DDLT, while the time for SLKT was longer by 80 minutes because renal transplantation was also required.

All the patients received general anesthesia. Two LDLT patients and one SLKT patient were intubated preoperatively in the ICU, while the other patients received propofol or midazolam, fentanyl, and remifentanil during induction. All patients were anesthetized using inhalation anesthetic agents after intubation (Table 3). We checked the anesthetic depth of the inhalation agents with BIS or PSI and adjusted it at 0.5-1 MAC (Minimum Alveolar Concentration). We controlled the intraoperative BIS and PSI values at 25-60 and 20-40, respectively.

We used inhalation anesthetic agents sevoflurane and desflurane (64\% vs. $35 \%$ in LDLT; $14 \%$ vs. $85 \%$ in DDLT). We administered propofol for induction in $82 \%$ of patients in small divided doses of $0.8-2 \mathrm{mg} / \mathrm{kg}$ according to blood pressure and midazolam $(0.05-0.2 \mathrm{mg} / \mathrm{kg})$ in $17 \%$ of patients. We used rocuronium for muscle relaxation in all patients, which we continuously administered in $68 \%$ of LDLT patients. We monitored the infusion rate at a median of 6 (3-8) $\mu \mathrm{g} / \mathrm{kg} / \mathrm{min}$ with TOF-Watch ${ }^{\circledR}$ and changed it as needed. Since we were using the train-of-four count, we recorded a few points for each patient but could not evaluate muscle relaxation.

Since there was a lot of bleeding and ascites, the water balance was relatively low $(+2.6-4.5 \mathrm{~mL} / \mathrm{kg} / \mathrm{h})$ in all patients but was relatively high in SLKT patients $(5.5 \mathrm{~mL} / \mathrm{kg} / \mathrm{h})$. The urine volume was maintained at $1.5 \mathrm{~mL} / \mathrm{kg} / \mathrm{h}$ in LDLT, and the rate of diuretic use (human atrial natriuretic peptide [hANP], furosemide) was high. The hANP was continuously administered during induction without dialysis in patients with a preoperative eGFR of 20-40. Furosemide was used as a single dose at the end of surgery in patients with a preoperative eGFR of 60-100. For postoperative management to facilitate renal function, we increased the infusion until diuresis while draining pleural effusion and ascites.

During infusion, the albumin dosage was $32 \%$ of the total infusion and transfusion volume (Fig. 1). During a blood transfusion, Red Blood Cells (RBCs), Fresh Frozen Plasma (FFP), and platelets were used in larger quantities in DDLT than in LDLT patients; however, the doses of FFP and platelets were adjusted depending on individual patients. 




Fig. (1). Proportion of total infusion and transfusion volumes (LDLT) $(n=51)$.

*RBC: red blood cell, **FFP: frozen fresh plasma, ***PC: platelet concentrates, Every item is the median of all the LDLT cases.

Table 3. Intraoperative management.

\begin{tabular}{|c|c|c|c|}
\hline Variable & LDLT & DDLT & SLKT \\
\hline Operation time (minutes) & $682(114)$ & $690(113)$ & $770(83)$ \\
\hline Anesthesia time (minutes) & $792(120)$ & $786(119)$ & $894(77)$ \\
\hline GRWR (\%) & $0.97(0.54-2.36)$ & $2.36(1.81-3.98)$ & $3.12(2.47-3.16)$ \\
\hline Cold ischemia time (minutes) & $137(87)$ & $448(111)$ & $335(92)$ \\
\hline Warm ischemia time (minutes) & $45(9)$ & $43(13)$ & $46(11)$ \\
\hline Intraoperative variables & - & - & - \\
\hline Water balance (in-out, $\mathrm{ml} / \mathrm{kg} / \mathrm{h}$ ) & $4.5(4.4)$ & $2.6(4.4)$ & $5.5(4.1)$ \\
\hline Urine volume $(\mathrm{ml} / \mathrm{kg} / \mathrm{h})$ & $1.5(1.1)$ & $0.5(0.1)$ & $0.2(0.5)$ \\
\hline Bleeding volume (L) & $2.8(0.4-22.0)$ & $6.9(3.0-25.2)$ & $4.0(1.1-14.1)$ \\
\hline Number of using diuretics (\%) & $16(31 \%)$ & $1(14 \%)$ & $5(100 \%)$ \\
\hline Number of using HES (\%) & $16(31 \%)$ & $1(14 \%)$ & $0(0 \%)$ \\
\hline RBC (U) & $8(0-62)$ & $12(8-68)$ & $18(8-36)$ \\
\hline FFP (U) & $0(0-40)$ & $8(0-43)$ & $2(0-44)$ \\
\hline $5 \%$ Albumin $(\mathrm{ml})$ & $2500(0-7000)$ & $2750(1750-8700)$ & $3750(3750-6250)$ \\
\hline Platelet (U) & $0(0-70)$ & $20(0-112)$ & $0(0-50)$ \\
\hline Dosage of adrenaline $(\mathrm{mg})$ & $0(0-0.5)$ & $0(0-0.03)$ & $0(0-0.04)$ \\
\hline Dosage of dopamine (mg) & $37(0-235)$ & $46(0-377)$ & $85(0-135)$ \\
\hline Dosage of noradrenaline $(\mathrm{mg})$ & $1.0(0-8.5)$ & $0.4(0-7.7)$ & $3.2(0.6-7.4)$ \\
\hline General anesthetic agent & - & - & - \\
\hline Sevoflurane N (\%) & $33(64 \%)$ & $1(14 \%)$ & $4(80 \%)$ \\
\hline Desflurane N (\%) & $18(35 \%)$ & $6(85 \%)$ & $1(20 \%)$ \\
\hline
\end{tabular}

Values are represented as mean (standard deviation), median (range, minimum-maximum), or number of patients (\%).

LDLT: living donor liver transplantation, DDLT: deceased donor liver transplantation, SLKT: simultaneous liver-kidney transplantation, GRWR: Graft-to-recipient body weight ratio, HES: Hydroxyethyl starch, RBC: red blood cell, FFP: fresh frozen plasma

Catecholamine was used to maintain the mean Arterial Blood Pressure (ABP) at 40-80 mmHg. Adrenaline was used in a few patients $(11 \%$ in LDLT, $14 \%$ in DDLT, $20 \%$ in SLKT), but dopamine and noradrenaline were used in all patients. On comparing the median values, the doses of dopamine and noradrenaline were found to be higher in SLKT patients (Table $\mathbf{3}$ ).

The value of graft-to-recipient weight ratio varied from 0.54 to $2.36($ median $=0.97)$.

\subsubsection{Relationship between Colloidal Solutions and Postoperative Renal Function}

The SALINHES fluid 6\% solution (Hydroxyethyl starch $70 / 0.5$ ) or VOLUVEN 6\% solution (Hydroxyethyl starch $130 / 0.4$ ) was used at $7-42 \mathrm{~mL} / \mathrm{kg}$ (median=0) for $31 \%$ of LDLT and $14 \%$ of DDLT patients. Except for one patient, $5 \%$ albumin was used in all patients. For albumin preparation, the severity of ascites and pleural effusion before surgery and total serum protein after induction of anesthesia were checked. Albumin preparation was started in patients with hypo- 
proteinemia, and the dose was adjusted during surgery by monitoring the albumin level and ascites development perioperatively. Although the cut-off value for hypoproteinemia was not clearly defined, it was administered at $<5$ $\mathrm{g} / \mathrm{dl}$ of total protein.

\subsection{Postoperative Course}

The patients' postoperative courses are shown in Table 4. The hospitalization period ascendingly increased in LDLT, DDLT, and SLKT patients, but the length of ICU stay was shortest in DDLT patients. Of 63, six patients died, and all underwent LDLT. Of these, four patients died within one year.

Postoperative dialysis was introduced in two LDLT patients, but it was not directly related to intraoperative management. Dialysis was withdrawn in four of five SLKT patients and all patients with preoperative hepatorenal syndrome. On the day after surgery, eGFR was maintained at the same value as what was compared with the rate before surgery in all transplantation methods, and it further improved at the time of discharge. The infection rate was $25-28 \%$ in LDLT and DDLT patients, whereas $0 \%$ in SLKT patients. The rejection rate was $23 \%$ in LDLT and $14 \%$ in DDLT patients, $0 \%$ in SLKT patients.

\subsection{Postoperative Intensive Care}

The patients were extubated at an average of a day after transplantation. There was no induction treatment with antibodies. The basic triple-drug regimen of immunosuppression was followed: tacrolimus, steroid, and Mycophenolate Mofetil (MMF). The trough level of tacrolimus was $7-9 \mathrm{ng} / \mathrm{dl}$ during the first month. Almost all patients with LDLT showed massive ascites. In order to avoid dehydration, the entire volume of drained ascites was meticulously compensated with crystalloid and 5\% albumin. The compensated volume was decreased until the amount of ascites decreased and graft growth was seen. It took 3-4 weeks for graft growth and the patients stayed in the ICU and HCU during this period of care.

\subsubsection{Characteristics of a Longer Stay in ICU and HCU}

We examined the length of ICU stay, including HCU stay as well. The mean length of stay was $28 \pm 21$ days in LDLT and $17 \pm 15$ days in DDLT patients. The characteristics of eight LDLT patients and one DDLT patient who stayed longer than the standard deviation were examined. Table $\mathbf{5}$ compares the preoperative, intraoperative, and postoperative conditions of patients with longer stays and other patients. Patients with longer stays tended to be older, and their primary disease was often alcoholic cirrhosis. The number of patients receiving preoperative dialysis was not high among long-term patients. Further, the operative time was long, intraoperative blood loss was high, and the amount of RBC transfusion and noradrenaline dose tended to be larger. The postoperative infection and reoperation rates were also higher.

Table 4. Postoperative course.

\begin{tabular}{|c|c|c|c|}
\hline Variable & $\begin{array}{c}\text { LDLT } \\
\mathrm{n}=51\end{array}$ & $\begin{array}{c}\text { DDLT } \\
\mathrm{n}=7\end{array}$ & $\begin{array}{c}\text { SLKT } \\
\mathbf{n}=5\end{array}$ \\
\hline Length of ICU and HCU stay (days) & $28(21)$ & $17(15)$ & $34(18)$ \\
\hline Length of hospitalization (days) & $66(33)$ & $71(25)$ & $78(55)$ \\
\hline Length of intubation (days) & $1(0-10)$ & $1(1-82)$ & $2(1-3)$ \\
\hline One-year mortality, N (\%) & $4(7 \%)$ & 0 & 0 \\
\hline Postoperative hemodialysis, N (\%) & $2(3 \%)$ & 0 & $1(20 \%)$ \\
\hline Postoperative infection, N (\%) & $13(25 \%)$ & $2(28 \%)$ & 0 \\
\hline Postoperative diabetes, N (\%) & $3(5 \%)$ & 0 & $1(20 \%)$ \\
\hline Graft failure, N (\%) & $1(1 \%)$ & 0 & 0 \\
\hline Rejection, N (\%) & $15(29 \%)$ & $1(14 \%)$ & 0 \\
\hline Reoperation, N (\%) & $11(21 \%)$ & 0 & $3(60 \%)$ \\
\hline Postoperative HbA1c (\%) & $5.8(1.4)$ & $5.6(0.4)$ & $4.7(0.2)$ \\
\hline eGFR $\left(\mathrm{ml} /\right.$ minute $\left./ 1.73 \mathrm{~m}^{2}\right)$ & - & - & - \\
\hline Post-operative day 1 & $69(38)$ & $50(16)$ & $21(17)$ \\
\hline At discharge & $65(33)$ & $60(33)$ & $96(34)$ \\
\hline
\end{tabular}

Values are represented in mean (standard deviation), median (range, minimum-maximum), or number of patients (\%).

LDLT: living donor liver transplantation, DDLT: deceased donor liver transplantation, SLKT: simultaneous liver-kidney transplantation, ICU: intensive care unit, eGFR: estimated glomerular filtration rates

Table 5. Preoperative patient characteristics (longer ICU- and HCU-stay cases)

\begin{tabular}{|c|c|c|}
\hline Variable & Longer Stay Cases & 9 \\
\hline Total patient number & $3(33 \%)$ & $25(46 \%)$ \\
\hline Male, N(\%) & $51.8(7.2)$ & $47.5(13.2)$ \\
\hline Age $($ years; average $)$ & $22.9(3.5)$ & $22.4(3.4)$ \\
\hline BMI $\left(\mathrm{kg} / \mathrm{m}^{2}\right)$ & & \\
\hline
\end{tabular}


(Table 5) contd.....

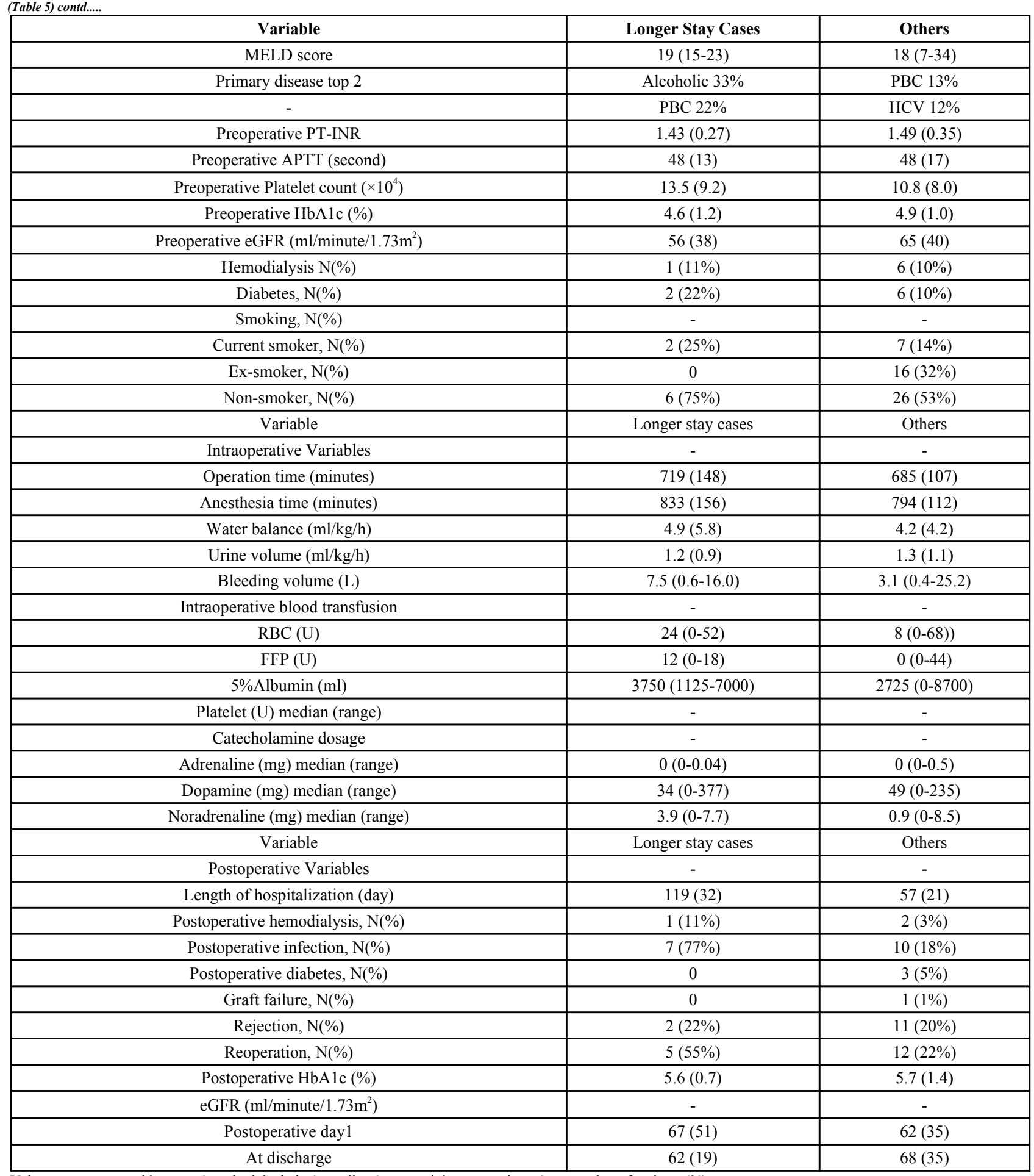

Values are represented in mean (standard deviation), median (range, minimum-maximum), or number of patients (\%).

LDLT: living donor liver transplantation, DDLT: deceased donor liver transplantation, SLKT: simultaneous liver-kidney transplantation, PT-INR: prothrombin time international normalized ratio, APTT: activated partial thrombin time, HbAlc: hemoglobin A1c, eGFR: estimated glomerular filtration rates

\section{DISCUSSION}

In this study, we made some major findings: (1) sevoflurane and desflurane were administered in the same way and both were useful as anesthetic agents for LT; (2) rocuronium was used at its usual dose despite liver failure because excessive bleeding results in unstable plasma drug concentration; (3) all patients undergoing preoperative dialysis due to acute kidney injury were successfully withdrawn from dialysis after surgery because appropriate transfusion management was expected to restore renal function; (4) the albumin infusion volume was over $30 \%$ of the total infusion and transfusion volume. Although albumin is expensive, we believe that it is reasonable to use it for maintaining the intravascular volume and osmotic pressure and protecting renal function.

Herein, we have discussed each evaluated item in detail. 


\subsection{Age}

The average patient age was 40 years. In recent times, the age of LT recipients in the USA has gradually increased; now, patients aged 50-64 years account for nearly half of all recipients (UNOS (United Network for Organ Sharing), https://optn.transplant.hrsa.gov/). The mean age is expected to increase if recipients with alcoholic cirrhosis and fatty liver increase in the future. LT for recipients with heart disease and diabetes mellitus will require further technical improvements and data accumulation.

\subsection{MELD Score}

In this study, the MELD score was approximately 18-19 (lowest $=7$; highest=34). Many SLKT patients had uncontrollable hepatic cyst infections due to ADPKD. Therefore, although the hepatic function was preserved, the MELD score in these patients remained high because of renal failure.

\subsection{Anesthetic Procedure}

All patients received general anesthesia, and the postoperative pain was controlled using fentanyl via intravenous patient-controlled analgesia (iv-PCA). Epidural anesthesia was not performed to avoid coagulation abnormality. Volatile anesthetic agents are hardly metabolized in the body, and their preconditioning effect has been reported to be organ-protective [2]. In this study, 38 patients received sevoflurane $(60 \%)$, and 25 received desflurane $(40 \%)$. In a randomized control trial comparing the incidence of Postreperfusion Syndrome (PRS) between sevoflurane and desflurane in LDLT, Lee et al. reported that PRS was significantly higher in the desflurane group (38.7 vs. 77.4, $\mathrm{p}=$ 0.004). Further, the desflurane group had heavier bleeding and used more epinephrine [3]. However, in another study, postoperative hepatorenal function and coagulability in LT donors were better with desflurane than with sevoflurane [4]. At our hospital, the anesthesiologist-in-charge determines the use of the inhalation agent according to each case, and thus, no clear criteria have been established. Since desflurane needs to be frequently filled in the vaporizer, some anesthesiologists avoid it.

Rocuronium was used as a muscle relaxant in all patients and was continuously administered to nearly $70 \%$ of patients. Many anesthesiologists perform continuous administration because they cannot afford to administer single doses frequently. Since rocuronium is metabolized in the liver, the continuous administration rate was increased or decreased using a muscle relaxation monitor. The dose used in our study was close to the usual dose (3-8 $\mu \mathrm{g} / \mathrm{kg} / \mathrm{min})$ to avoid excessive bleeding that could result in unstable plasma drug concentration.

\subsection{Intraoperative Monitoring}

A pulmonary artery catheter was inserted in 2 patients (3\%) with pulmonary hypertension. In other cases, cardiac output was estimated and managed only with ABP and FloTrac. TEE was useful not only for cardiac function assessment but also for infusion/transfusion volume load management. The American Society of Anesthesiologists also recommends TEE during LT. However, patients with liver failure often have esophageal varices, and TEE cannot be performed in such patients. In this study, TEE was used in $22 \%$ of patients. In one patient, it was difficult to extubate on the day after surgery due to lingual hematoma caused by TEE complications.

The BIS and Sedline were used as EEG monitors during anesthesia. Although there have been many reports about Postoperative Cognitive Dysfunction (POCD) with BIS-guided anesthesia [5 - 7], few studies have been conducted in patients with LT [8]. In this study, the delivery of anesthetic agents was managed by dose adjustment based on the intraoperative BIS/PSI values in all patients. POCD was found in 1 of 63 patients; however, it was described subjectively only in the medical record, and no delirium score was used. In many cases, $\mathrm{rSO}_{2}$ could not be measured due to jaundice associated with hyperbilirubinemia.

\subsection{Renal Function}

In end-stage liver failure, the Renin-AngiotensinAldosterone System (RAAS) is enhanced and is likely to cause the hepatorenal syndrome. In this study, all patients undergoing preoperative dialysis due to acute kidney injury were successfully withdrawn from dialysis after surgery, as appropriate transfusion management and the use of hANP were expected to restore renal function.

Regarding intraoperative diuretic use, hANP was continuously administered to patients with decreased renal function and self-urination, and furosemide was used in patients with decreased urine volume despite the maintenance of renal function. hANP promotes natriuresis by dilating afferent arterioles in the kidneys, increasing renal blood flow, and suppressing RAAS hormones. RAAS is enhanced in patients with terminal cirrhosis due to decreased renal blood flow; the use of hANP antagonizes this condition.

\subsection{Circulation}

Patients with terminal cirrhosis develop ascites and pleural effusion due to hypoalbuminemia and decreased effective circulating blood volume. Peripheral vascular resistance decreases due to dysfunction of endothelin and nitrous oxide and is further reduced by general anesthetic agents, which increases the risk of circulatory collapse. Additionally, coagulation disorders cause intraoperative blood loss, and hypotension is likely to occur during surgery after anesthesia induction. The use of catecholamines is essential to combat this.

Hypotension during LT can affect the induction of anesthesia, drainage of ascites, clumping of the Inferior Vena Cava (IVC), and reperfusion. It is important to communicate with surgeons at each time point and control the dosage of catecholamines. In many of our patients, catecholamines (noradrenaline and dopamine) were continuously administered after central venous access was secured. In 3 patients with less blood loss, the condition was controlled only with single doses of ephedrine and phenylephrine. However, it is difficult to predict intraoperative hemodynamics in LT, and it was considered reasonable to start continuous catecholamine 
administration in small amounts to correspond to hypotension immediately, even in patients with no hypotension at the time of anesthesia induction. Especially during the IVC-clumping period, catecholamines $(0.05-0.3 \mathrm{mcg} / \mathrm{kg} / \mathrm{min}$ of noradrenaline and $3-5 \mathrm{mcg} / \mathrm{kg} / \mathrm{min}$ of dopamine) were mainly used for maintaining blood pressure rather than just increasing infusion. Their dosage was based on cardiac output, cardiac index, and a mean ABP of at least $40 \mathrm{mmHg}$. Avoiding perfusion of excess fluid could protect the donor's liver and reduce respiratory complications. In some DDLT patients, adrenaline was required because abnormal hypotension due to reperfusion injury may have occurred if the duration of ischemia in the donor's liver was prolonged. No complications caused by intraoperative hypotension were observed.

\subsection{Infusion/Transfusion}

The colloid infusions, 5\% albumin and HES, were used (except for one patient in whom albumin was not used). If the colloid osmotic pressure did not improve after surgery, the intubation period was prolonged to avoid pulmonary edema or pleural effusion. Enough amount of albumin was used to significantly improve the patients' lung condition. The albumin infusion volume was over $30 \%$ of the total infusion and transfusion volume (Fig. 1). Although albumin infusions are expensive, they might be reasonable for maintaining intravascular volume, osmotic pressure, and protecting renal function. For RBC transfusion, a necessary volume was transfused while checking blood pressure, Hemoglobin $(\mathrm{Hb})$, and Hematocrit $(\mathrm{Ht})$. Since high $\mathrm{Ht}$ increases the risk of thrombosis, RBC was administered to maintain a volume of around $30 \%$. Further, the hemostatic coagulation system gets disrupted in end-stage liver disease. Therefore, bleeding and embolism were likely to occur. It has been reported that transfusion of FFP and platelets are more likely to cause portal vein thrombosis [9]. Therefore, in our hospital, we try not to transfuse FFP or platelets as far as possible, as was the case in this study.

\subsubsection{Blood Glucose Level}

In patients with chronic liver failure, reduced glycogen storage and gluconeogenesis may easily cause hypoglycemia. However, insulin resistance is decreased, which often results in abnormal glucose tolerance.

In this study, preoperative $\mathrm{HbAlc}$ levels were within the normal range but tended to increase after surgery. The onset of postoperative diabetes was also observed in 5\% of LDLT patients. In LT surgery, blood glucose levels greatly fluctuate due to intraoperative stress and steroid use. A systematic review reported that the mortality and infection rates increase when the blood glucose level is $\geq 150 \mathrm{mg} / \mathrm{Dl}$, and the organ rejection rate increases when the blood glucose level is $\geq 200$ $\mathrm{mg} / \mathrm{dL}[10]$. Although there is no literature on intraoperative blood glucose levels and outcomes, it is reasonable to believe that intraoperative blood glucose control positively affects outcomes.

\subsubsection{Prolonged ICU and HCU Stays}

The characteristics of eight LDLT patients and one DDLT patient who stayed relatively longer than the standard deviation was examined (Table 5). In our hospital, patients with exceptionally long ICU stays were older and had alcoholic cirrhosis as the primary disease, which is in line with previous reports $[11,12]$. The infection and reoperation rates were also high because many patients contracted intraperitoneal infections or underwent reopening with lavage drainage. Patients with prolonged stays also tended to have longer operation times and larger amounts of bleeding. Difficult surgery may have also affected the postoperative outcome. However, these details were not detected thoroughly because the medical records were analyzed retrospectively.

Our study had some limitations. Firstly, the retrospective nature of the study did not permit us to set objective endpoints, such as postoperative infection rate. Secondly, the total number of patients who underwent DDLT and SLKT was small. Because the number of these target cases is small, even if clinically useful conclusions are shown, it might not be a convincing data analysis result to be included in the guideline.

\section{CONCLUSION}

The anesthetic management of LT was conducted empirically in our study, during which the 5 -year survival rate was above average ( $88 \%$ in LDLT patients). Based on our results, we concluded that anesthetic management of LT surgery should be adapted to each patient's case. Although we were not able to propose an optimal anesthetic method, we depicted the current status and direction of intraoperative management in LT surgery. Based on our results, we drew some conclusions; first, the usual dose of rocuronium is required because excessive bleeding may result in unstable plasma drug concentration; second, an adequate amount of albumin should be used and transfusion of FFP or platelets should be avoided as far as possible because of excessive thrombosis; third, the use of hANP (human atrial natriuretic peptide) drug infusion and appropriate transfusion management can be expected to restore renal function. Since there are various surgical approaches and surgeons' preferences may differ between institutions, large-scale multi-institutional studies or meta-analyses are necessary to further explore this topic in the future.

\section{LIST OF ABBREVIATIONS}

$\begin{array}{ll}\text { ABP } & =\text { Arterial Blood Pressure } \\ \text { ADPKD } & =\text { Autosomal Dominant Polycystic Kidney Disease } \\ \text { BIS } & =\text { Bispectral Index } \\ \text { DDLT } & =\text { Deceased Donor Liver Transplantation } \\ \text { FFP } & =\text { Fresh Frozen Plasma } \\ \text { GRWR } & =\text { Graft-to-recipient Body Weight Ratio } \\ \text { hANP } & =\text { Human Atrial Natriuretic Peptide } \\ \text { Hb } & =\text { Hemoglobin } \\ \text { HCU } & =\text { High Care Unit } \\ \text { HES } & =\text { Hydroxyethyl Starch } \\ \text { Ht } & =\text { Hematocrit } \\ \text { ICU } & =\text { Infensive Care Unit } \\ \text { IVC } & =\text { Intravenous Patient-Controlled Analgesia } \\ \text { iv-PCA } & =\text { Living Donor Liver Transplantation } \\ \text { LDLT } & =\text { Liver Transplantation } \\ \text { LT } & \end{array}$




$\begin{array}{ll}\text { MAC } & =\text { Minimum Alveolar Concentration } \\ \text { MELD score } & =\text { Model for End-Stage Liver Disease Score } \\ \text { MMF } & =\text { Mycophenolate Mofetil } \\ \text { PBC } & =\text { Primary Biliary Cholangitis } \\ \text { PRS } & =\text { Postreperfusion Syndrome } \\ \text { PSI } & =\text { Patient State Index } \\ \text { RAAS } & =\text { Renin-Angiotensin-Aldosterone System } \\ \text { RBCs } & =\text { Red Blood Cells } \\ \text { SLKT } & =\text { Simultaneous Liver-Kidney Transplantation }\end{array}$

\section{AUTHORS' CONTRIBUTIONS}

All authors contributed to the study's conception and design. Material preparation, data collection, and analysis were performed by $\mathrm{HH}$ and $\mathrm{MO}$. The first draft of the manuscript was written by $\mathrm{HH}$, and all the authors commented on previous versions of the manuscript. All the authors read and approved the final manuscript.

\section{ETHICS APPROVAL AND CONSENT TO PARTI- CIPATE}

This study was approved by the ethics committee of Clinical and Academic Research Promotion Center of Tokyo Women's Medical University with approval number: 5051, the date of obtainment was January 17, 2019.

\section{HUMAN AND ANIMAL RIGHTS}

No animals were used in this research. All human research procedures followed were in accordance with the ethical standards of the committee responsible for human experimentation (institutional and national), and with the Helsinki Declaration of 1975, as revised in 2013.

\section{CONSENT FOR PUBLICATION}

Patients provided consent using the opt-out method. Written informed consent was obtained as much as possible.

\section{STANDARDS OF REPORTING}

STROBE guidelines were followed for this study.

\section{AVAILABILITY OF DATA AND MATERIALS}

The datasets analyzed during the current study are available from the corresponding author on reasonable request.

\section{FUNDING}

None.

\section{CONFLICT OF INTEREST}

The authors declare no conflict of interest, financial or otherwise.

\section{ACKNOWLEDGEMENTS}

We greatly appreciate the cooperation of the members of the clinical research, innovation, and education center of Tokyo Women's Medical University. We are also grateful to all the investigators in this study, especially Yasuko Nagasaka (Chair of Department of Anesthesiology, Tokyo Women's Medical University, Tokyo, Japan), Masakazu Yamamoto, Akiko Omori, and Takaaki Kato (Department of Gastroenterology, Tokyo Women's Medical University, Tokyo, Japan).

\section{REFERENCES}

[1] The Japan Society for Transplantation. Organ transplantation seen in data. Available from: http://www.asas.or.jp/jst/general/number

[2] Beck-Schimmer B, Breitenstein S, Urech S, et al. A randomized controlled trial on pharmacological preconditioning in liver surgery using a volatile anesthetic. Ann Surg 2008; 248(6): 909-18.

[http://dx.doi.org/10.1097/SLA.0b013e31818f3dda] [PMID: 19092335]

[3] Lee J, Yoo YJ, Lee JM, Park YJ, Ryu HG. Sevoflurane versus desflurane on the incidence of postreperfusion syndrome during living donor liver transplantation: A randomized controlled trial. Transplantation 2016; 100(3): 600-6.

[http://dx.doi.org/10.1097/TP.0000000000000874] [PMID: 26335917]

[4] Ko JS, Gwak MS, Choi SJ, et al. The effects of desflurane and sevoflurane on hepatic and renal functions after right hepatectomy in living donors. Transpl Int 2010; 23(7): 736-44.

[http://dx.doi.org/10.1111/j.1432-2277.2009.01050.x]

[PMID: 20102552]

[5] Radtke FM, Franck M, Lendner J, Krüger S, Wernecke KD, Spies CD. Monitoring depth of anaesthesia in a randomized trial decreases the rate of postoperative delirium but not postoperative cognitive dysfunction. Br J Anaesth 2013; 110(Suppl. 1): i98-i105.

[http://dx.doi.org/10.1093/bja/aet055] [PMID: 23539235]

[6] Chan MT, Cheng BC, Lee TM, Gin T. BIS-guided anesthesia decreases postoperative delirium and cognitive decline. J Neurosurg Anesthesiol 2013; 25(1): 33-42.

[http://dx.doi.org/10.1097/ANA.0b013e3182712fba] [PMID: 23027226]

[7] Ballard C, Jones E, Gauge N, et al. Optimised anaesthesia to reduce post operative cognitive decline (POCD) in older patients undergoing elective surgery, a randomised controlled trial. PLoS One 2012; 7(6)e 37410

[http://dx.doi.org/10.1371/journal.pone.0037410] [PMID: 22719840]

[8] Aceto P, Perilli V, Lai C, Ciocchetti P, Vitale F, Sollazzi L. Postoperative cognitive dysfunction after liver transplantation. Gen Hosp Psychiatry 2015; 37(2): 109-15.

[http://dx.doi.org/10.1016/j.genhosppsych.2014.12.001] [PMID: 25550172]

[9] Gimeno FA, Calvo J, Loinaz C, et al. Comparative analysis of the results of orthotopic liver transplantation in patients with and without portal vein thrombosis. Transplant Proc 2005; 37(9): 3899-903.

[http://dx.doi.org/10.1016/j.transproceed.2005.10.085] [PMID: 16386578]

[10] Paka P, Lieber SR, Lee RA, Desai CS, Dupuis RE, Barritt AS. Perioperative glucose management and outcomes in liver transplant recipients: A qualitative systematic review. World J Transplant 2018; 8(3): 75-83.

[http://dx.doi.org/10.5500/wjt.v8.i3.75] [PMID: 29988867]

[11] Stratigopoulou P, Paul A, Hoyer DP, Kykalos S, Saner FH, Sotiropoulos GC. High MELD score and extended operating time predict prolonged initial ICU stay after liver transplantation and influence the outcome. PLoS One 2017; 12(3)e0174173

[http://dx.doi.org/10.1371/journal.pone.0174173] [PMID: 28319169]

[12] Gacouin A, Legay F, Camus C, et al. At-risk drinkers are at higher risk to acquire a bacterial infection during an intensive care unit stay than abstinent or moderate drinkers. Crit Care Med 2008; 36(6): 1735-41. [http://dx.doi.org/10.1097/CCM.0b013e318174dd75] [PMID: 18520640] 